\title{
The Theory of Critical Distances applied to multiscale toughening mechanisms
}

\author{
David Taylor \\ Department of Mechanical \& Manufacturing Engineering, Trinity College Dublin, The University of Dublin, Dublin 2, Ireland
}

\section{A R T I C L E I N F O}

\section{Keywords:}

TCD

Critical distance

Toughness

Short crack

Bone

\begin{abstract}
A B S T R A C T
The Theory of Critical Distances (TCD) is an approach used for predicting the effect of cracks and notches on fracture and fatigue behaviour. In recent years it has been successfully applied to a wide range of problems across all classes of materials. The approach relies on the identification of a material constant known as the critical distance, $L$, which can only be determined by fitting predictions to experimental data. It has been argued that $L$ is related to the size of microstructural features which affect crack growth, but this argument becomes problematic in the presence of multiple toughening mechanisms operating at different scales. In the present work, this matter was approached through the use of thought experiments conducted on model microstructures. It was found that if two mechanisms operate at different length scales, then the use of the normal TCD approach is still possible, though accuracy is reduced and the value of $L$ obtained falls between the length scales of the two mechanisms. A more sophisticated approach however can successfully identify two relevant $L$ values and thus provide insights into the underlying mechanisms. A practical example is given: the analysis of short and long crack toughness data for bone, for which mechanisms at the hundred-micron and millimetre scales were successfully identified. Some general conclusions emerge about the effect of hierarchical toughening mechanisms: in particular it was found that the mechanisms operating at the small length scales may control the strength of the material but have relatively little effect on its toughness.
\end{abstract}

\section{Introduction}

Fracture mechanics has developed to the point where we are able to characterise the toughness and fatigue strength of materials very accurately based on experiments. But considerable challenges remain when it comes to the underlying physical mechanisms which control these parameters. Thanks to advances in testing, microscopy and computer simulation we are now able to identify and model those processes which hinder or encourage crack propagation, allowing toughening mechanisms to be elucidated [1-3]. However it is still proving difficult to quantify these effects, especially when several mechanisms may operate simultaneously. Better understanding in this area would allow us to design materials with improved toughness and better fatigue behaviour in a more systematic manner.

The Theory of Critical Distances (TCD) is an approach for predicting the effect of stress concentration features in failure modes where cracking occurs, such as brittle fracture and fatigue [4]. It has been successfully used for a wide range of cases, including notches, holes, short cracks and contact problems. It has been applied to all classes of materials: recent examples include polymers [5] composites [6], rocks [7], welded joints [8] low-cycle fatigue [9] and fretting fatigue [10]. Within the general TCD approach there are several different methods, all of which require the definition of a material parameter with dimensions of length: the critical

E-mail address: dtaylor@tcd.ie. 


\section{Nomenclature}

a crack length

ai initial crack length

d microstructural distance (e.g. grain size)

$\mathrm{d}_{\text {macro }} \quad$ the larger of two microstructural distances

$\mathrm{d}_{\text {micro }}$ the smaller of two microstructural distances

$\delta \quad$ crack opening displacement

$\delta_{\mathrm{b}} \quad$ critical crack opening displacement for ligament failure

Sa crack growth increment

$\Delta \sigma_{\mathrm{o}} \quad$ fatigue limit

$\Delta \mathrm{K}_{\text {th }} \quad$ fatigue crack propagation threshold

E Young's modulus

$\mathrm{K} \quad$ stress intensity

$\mathrm{K}_{\mathrm{b}} \quad$ critical stress intensity to overcome a barrier

$\mathrm{K}_{\mathrm{c}} \quad$ fracture toughness

$\mathrm{K}_{\mathrm{cm}} \quad$ measured fracture toughness (for different initial crack lengths)

$\begin{array}{ll}\mathrm{K}_{\mathrm{cmacro}} & \begin{array}{l}\text { fracture toughness due to larger microstructural } \\ \text { feature } \\ \text { fracture toughness due to smaller microstructural } \\ \mathrm{K}_{\mathrm{cmicro}}\end{array} \\ \mathrm{K}_{\mathrm{i}} & \begin{array}{l}\text { feature } \\ \text { initiation toughness } \\ \mathrm{K}_{\mathrm{m}}\end{array} \\ \mathrm{K}_{\mathrm{net}} & \begin{array}{l}\text { fracture toughness of the matrix material } \\ \mathrm{L}\end{array} \\ \mathrm{P} & \text { critical distance } \\ \mathrm{P}_{\mathrm{factor}} & \text { force in a stretched fibre/ligament } \\ \mathrm{PM} & \text { Point Method } \\ \mathrm{r} & \text { distance ahead of crack tip } \\ \mathrm{r}_{\mathrm{i}} & \text { residual (error in predicting ith value of of) } \\ \sigma & \text { stress } \\ \sigma_{\mathrm{f}} & \text { fracture stress } \\ \sigma_{\mathrm{p}} & \text { predicted value of fracture stress } \\ \sigma_{\mathrm{u}} & \text { material ultimate strength } \\ \mathrm{TCD} & \text { Theory of Critical Distances } \\ \mathrm{x} & \text { distance from crack centre }\end{array}$

distance $L$. Stress-based methods involve the determination of a characteristic stress, defined as the stress at a given distance $(L / 2)$ from the notch root or averaged over a region ahead of the notch whose size depends on $L$. Stress-intensity based methods include the insertion of an imaginary crack (of length $L$ ) or the assumption of a step-wise mode of crack extension in which the step size is $2 L$ [11]. Alternative methods (often referred to by the term "Finite Fracture Mechanics") involve using the stress and stress intensity (i.e. energy) methods simultaneously whilst dropping the constant- $L$ assumption [12,13].

The value of $L$ cannot be known a priori: it must be determined by carrying out experiments, for example using specimens containing notches of different lengths or root radii. A frequent point of discussion when using the TCD is whether or not the value of

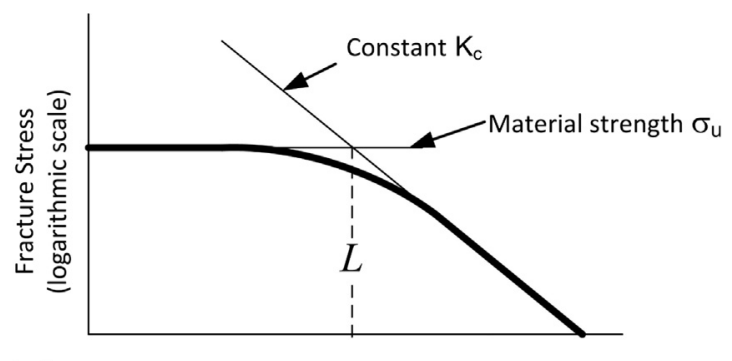

(a)

$$
\text { Crack Length (logarithmic scale) }
$$

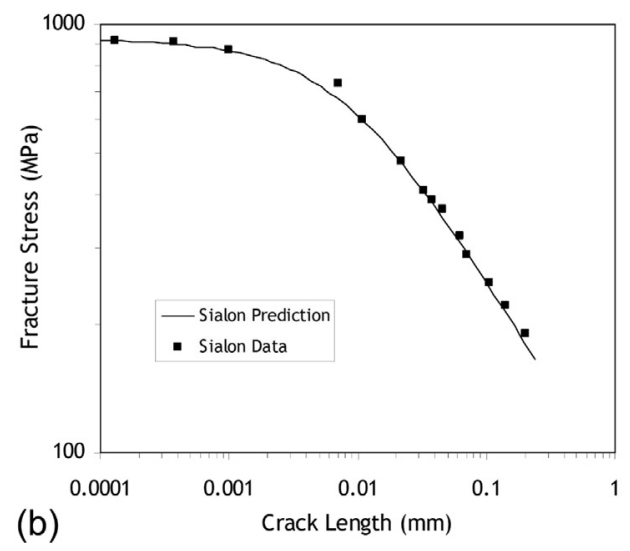

Fig. 1. (a) Schematic showing the effect of crack length on fracture stress. Experimental data tend to lie on a curve (thick line) conforming to a constant stress $\sigma_{\mathrm{u}}$ at small $a$ and a constant stress intensity $K_{\mathrm{c}}$ at large $a$. The critical distance $L$ corresponds to the intersection of the straight lines describing these two extremes. (b) An example of experimental data of this kind (a Sialon material [22]) which was accurately predicted using the TCD approach [16]. 
$L$ thus obtained has any physical significance. For example, can $L$ be related to some parameter in the microstructure of the material, and thus provide insights into the physical mechanisms of crack growth and toughening? In a few cases this link can be clearly made. For example when the data on brittle cleavage fracture in notched samples of steel with different grain sizes was examined, it was found that the value of $L$ was always equal to the grain size $d$ [14]. This could be explained in terms of the well-known RKR mechanism [15] whereby failure occurs as a result of the unstable propagation of a cracked carbide. Since these carbides are located in the grain boundaries the nearest one will be, on average, a distance of $d / 2$ from the notch root.

In other cases correlations appear which are suggestive but require further analysis. For example the consideration of short-crack data from a range of engineering ceramic materials showed that $L$ had an average value of 5 times the grain size [16,17], suggesting the role of mechanisms operating at the scale of the grain but involving more than one grain, such as intergranular microcracks ahead of the crack or grain face interaction behind the crack. In other cases the value of $L$ does not appear to correspond to any microstructural scale: for example in fibre composite laminates $L$ usually has a value of several millimetres, much larger than any structural unit [18], and the brittle polymer PMMA has an $L$ value of $100 \mu \mathrm{m}$ despite being amorphous and therefore having no microstructure [19].

In previous work [20,21] I developed a novel approach to investigate this problem through the use of thought experiments conducted on model microstructures. The approach was to imagine a material having a simplified microstructure which displayed one of the well-known toughening mechanisms, to deduce the fracture data for this material from first principles and then to use the TCD to predict this data. $L$ was found to be proportional to some microstructural distance $d$ in all cases, though with widely differing constants of proportionality, showing that simply equating $L$ with the size of a known microstructural feature is not always appropriate.

Materials often have structure at various different length scales: atomic/molecular structure at the nanometre scale, microstructure at various scales in the micron to hundred micron range and macrostructure at the millimetre scale or larger. Structural features of different sizes may all contribute to facilitate or hinder crack propagation. But if there are two or more mechanisms operating at different length scales, can the TCD approach still be used to predict the results, and if so what will be the resulting value of $L$ ? Can the TCD be modified in some way to take account of multiple toughening mechanisms?

The work described here addresses these questions using the model material approach developed previously, now extended to include materials with multiple mechanisms. It includes a case study using experimental data from bone, which is a material that is known to display structure, and toughening mechanisms, at different length scales.

\section{The short crack problem}

A well known problem in fracture mechanics concerns the effect of crack length on fatigue and fracture behaviour. Fig. 1a illustrates the problem schematically. Tests to measure the fracture stress $\sigma_{\mathrm{f}}$ for a series of specimens containing cracks of different
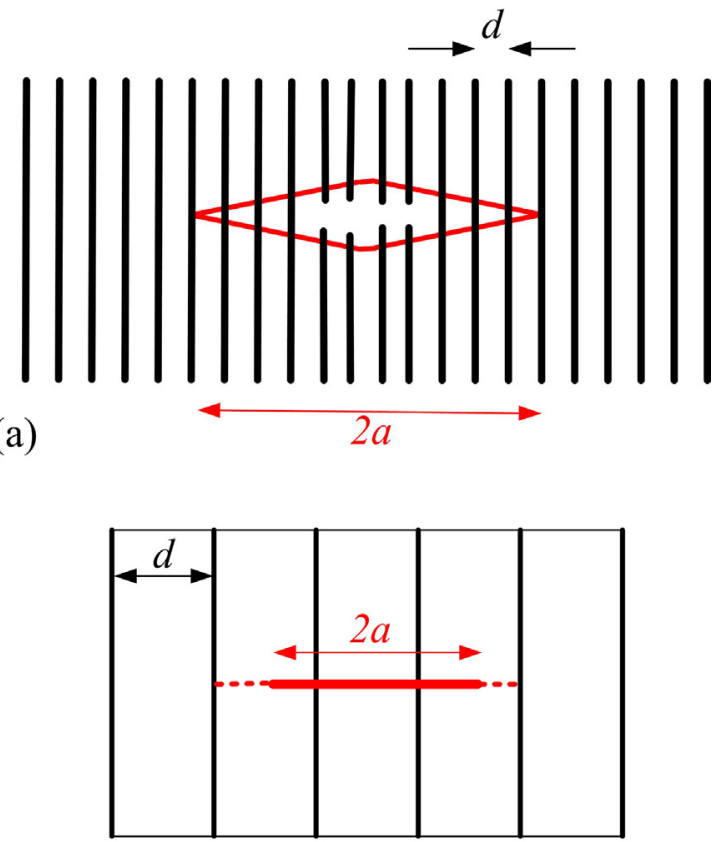

(b)

Fig. 2. Illustrations of the two model materials, showing in each case a centre-crack of length $2 a$. (a) The ligament model, having fibres of separation $d$ : unbroken fibres just behind the crack tip constitute a process zone. A force P acts in each fibre, tending to close the crack. (b) The barriers model, having barriers of separation $d$. 
length $a$ should fall on a straight line of slope -0.5 on a $\log / \log$ plot, in order to conform to the standard equation (stated here for a centre crack in tension):

$$
K_{c}=\sigma_{f} \sqrt{(\pi a)}
$$

where $K_{c}$ is the material's fracture toughness. However this relationship must break down as $a \rightarrow 0$ because otherwise $\sigma_{\mathrm{f}}$ would rise to infinity. In practice the maximum possible value of $\sigma_{\mathrm{f}}$ is the material's strength $\sigma_{\mathrm{u}}$ and experimental data tend to lie on a curved line as shown. Examples of this kind of data are the effect of small defects in engineering ceramic materials [22,23] and fibre composites $[18,24]$. The same type of problem arises in fatigue, where $\sigma_{\mathrm{f}}$ becomes the fatigue limit stress range $\Delta \sigma_{\mathrm{o}}$ and $K_{\mathrm{c}}$ becomes the fatigue crack propagation threshold $\Delta K_{\text {th }}[25,26]$. This is an important problem because many engineering components fail from small fatigue cracks, manufacturing defects, inclusions etc.

The short crack problem will be used in the present paper because it is a good example of a problem which can be addressed using the TCD. As Fig. 1b shows, experimental data often conform to a TCD prediction. In earlier work using model materials, I showed that their short crack behaviour could also be predicted accurately using the TCD. The problem is thus suitable for extension to the case of model materials with multiple toughening mechanisms.

\section{Methods}

The effect of crack length on failure stress will be investigated in two model materials. The first, which will be referred to as the "ligaments model" is illustrated in Fig. 2a. This is a simplified version of a toughening mechanism which occurs in various materials, including long-fibre composites and PMMA. It consists of parallel fibres of spacing $d$, embedded in a matrix. The crack, assumed to be a centre crack of half-length $a$ in an infinite plate, oriented with its plane normal to the fibres, grows through the matrix material, leaving unbroken fibres behind the crack tip. These fibres act as supporting ligaments, reducing the stress intensity. If the crack opening displacement $\delta$ exceeds a critical value $\delta_{\mathrm{b}}$ the fibre breaks and no longer affects the stress intensity. As a result, there are a certain number of unbroken fibres immediately behind the crack tip which constitute a process zone. The force $P$ in a fibre is assumed to be proportional to the crack opening displacement, thus:

$$
P=P_{\text {factor }} \delta
$$

Some standard fracture mechanics solutions can be used $[27,28] ; \delta$ is related to the applied stress intensity $K$, material Young's modulus $E$, and distance from the crack centre $x$ as [28]:

$$
\delta=\frac{2 K}{E} \sqrt{\frac{a^{2}-x^{2}}{\pi a}}
$$

The (negative) K contributed by a given fibre which exerts a force $P$ at a distance $x$ is [27]:

$$
K=\frac{2}{\pi}\left[\frac{1+f(x / a)}{\sqrt{a^{2}-x^{2}}}\right] P \sqrt{\pi a}
$$

where:

$$
f(x / a)=\left[1-\left(\frac{x}{a}\right)^{2}\right]\left\{0.2945-0.3912\left(\frac{x}{a}\right)^{2}+0.7685\left(\frac{x}{a}\right)^{4}-0.9942\left(\frac{x}{a}\right)^{6}+0.5094\left(\frac{x}{a}\right)^{8}\right\}
$$

The initial crack is assumed to have zero tip radius, and is introduced into the material in such a way that it initially has no process zone. So it might more accurately be referred to as a perfectly sharp notch or slot. The effect of other types of initial crack will be discussed below. The crack tip is initially located a distance $d / 2$ from the nearest fibre (as this will be the average position if the crack is introduced at a random location). The crack will grow provided its stress intensity is greater than the fracture toughness of the matrix, $K_{m}$. As the crack encounters fibres they will act to reduce $K$, causing crack arrest when $K$ falls below $K_{m}$. The fracture stress of the material is defined as the lowest stress that can be applied which will allow crack growth.

Two versions of this material are defined: a "micro" version in which the spacing is $d_{\text {micro }}=1$ and a "macro" version having $d_{\text {macro }}=100$. Table 1 shows the values of the various constants for the two models: note that the units of all quantities are unstated since they are arbitrary. Since the aim is to compare relative values of derived quantities (such as fracture strength), rather than to derive their absolute values, the system of units used will have no effect on the results. Material constants $\mathrm{K}_{\mathrm{m}}$ and $\mathrm{E}$ are unchanged, whilst constants relating to the behaviour of the fibres $\left(\mathrm{P}_{\text {factor }}\right.$ and $\left.\delta_{\mathrm{b}}\right)$ are different in the micro and macro models. These constants have been chosen so that the long-crack fracture toughness values for the micro and macro models are $K_{\text {cmicro }}=1.0$ and $K_{\text {cmacro }}$

Table 1

Values of constants used in the ligament models.

\begin{tabular}{llllll} 
& $\mathrm{d}$ & $\mathrm{E}$ & $\mathrm{K}_{\mathrm{m}}$ & $\mathrm{P}_{\text {factor }}$ & $\delta_{\mathrm{b}}$ \\
\hline Micro & 1 & 1 & 0.5 & 0.01 & 10.3 \\
Macro & 100 & 1 & 0.5 & 0.0165 & 325 \\
\hline
\end{tabular}


$=\sqrt{ } 10=3.16$. These values were chosen because there is an established relationship linking the critical distance $\mathrm{L}$ to the ultimate strength and the fracture toughness [4]:

$$
L=\frac{1}{\pi}\left(\frac{K_{c}}{\sigma_{u}}\right)^{2}
$$

Since the structural unit size $d$ for the micro and macro models differs by a factor of 100 , then if their $\mathrm{K}_{\mathrm{c}}$ values differ by $\sqrt{ } 10$ one would expect their $\sigma_{\mathrm{u}}$ values also to vary by $\sqrt{ } 10$ (with the micro one being larger) if the TCD applies. The constants were also chosen so that, in both micro and macro models, the size of the process zone for a long crack at an applied stress intensity $K_{\mathrm{c}}$ would be 40 times the fibre spacing, giving rise to 40 unbroken ligaments.

The second material model is referred to as the "barriers model". Examples of microstructural barriers to crack growth are grain boundaries in metals and osteons in bone. As shown in Fig. 2b, the material has a series of parallel barriers, of spacing $d$. The stress intensity required for a crack to pass through a barrier is $\mathrm{K}_{\mathrm{b}}$ : the toughness of the intervening matrix material is assumed to be negligible. The type and location of the initial crack are the same as in the ligaments model. Micro and macro versions of this model are defined in a similar manner to those in the ligaments model, so $d_{\text {micro }}=1$ and $d_{\text {macro }}=100$ and the resulting long-crack toughness values are $K_{\text {cmicro }}=1.0$ and $K_{\text {cmacro }}=\sqrt{ } 10=3.16$. Again, fracture stress is found as the lowest stress to ensure continued propagation of the crack. This can be found by noting that, since the initial crack tip is always a distance $d / 2$ from the first barrier, and since the intervening material is assumed to have a negligible toughness, then as soon as any stress is applied the crack will increase its length by $d / 2$. Since the measured toughness value $\mathrm{K}_{\mathrm{cm}}$ is calculated using the initial crack length only, it will thus be related to the barrier toughness by:

$$
\frac{K_{c m}}{K_{b}}=\sqrt{\frac{a_{i}}{a_{i}+d / 2}}
$$

These two models represent, in a simplified manner, two mechanisms which are known to act in real materials. Here, by using
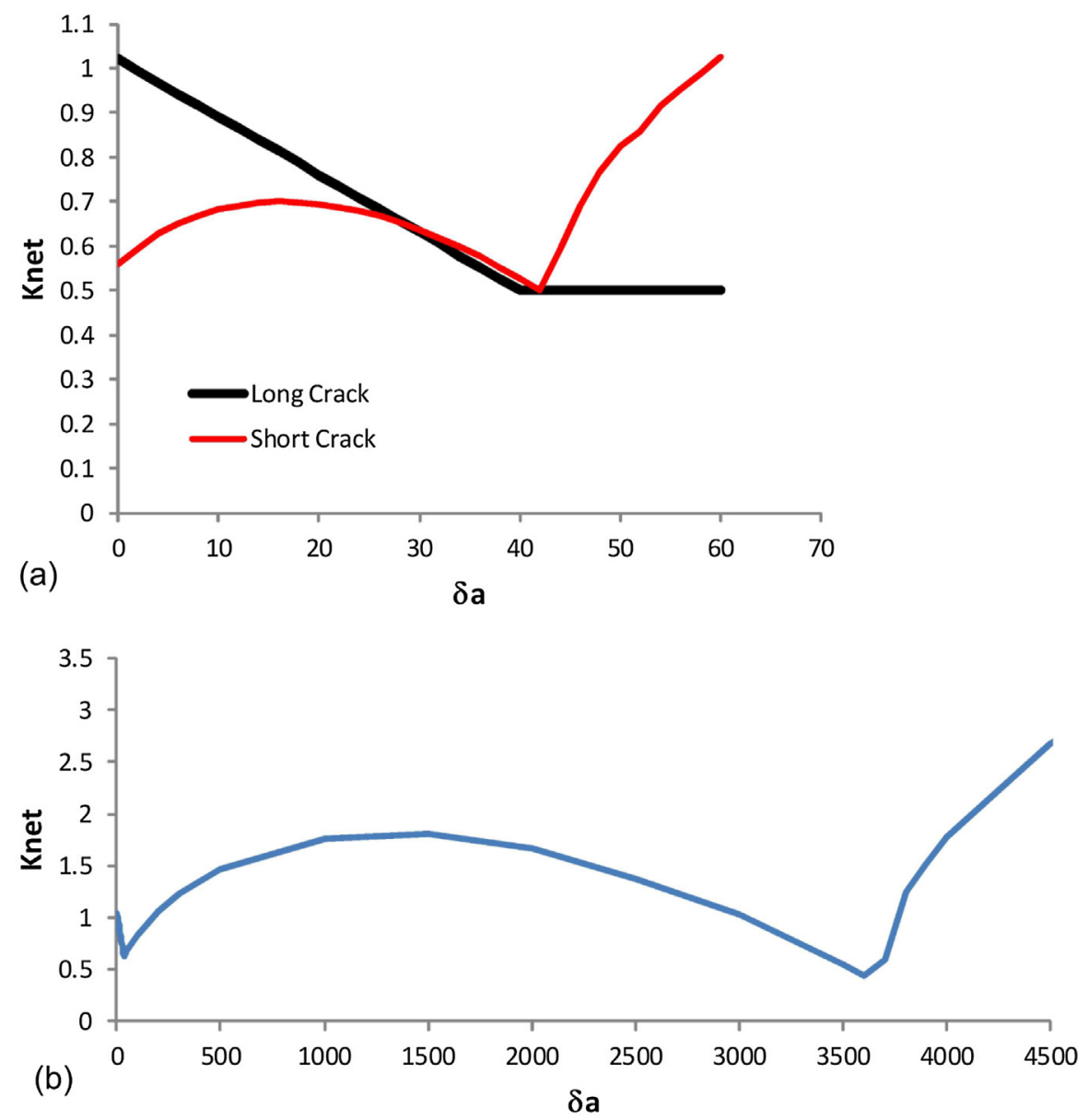

Fig. 3. Results from the ligaments models, showing the variation of the overall stress intensity $\mathrm{K}_{\text {net }}$ with crack extension $\delta a$ for applied stress equal to the predicted fracture stress in each case. (a) The model with micro ligaments only, for a long crack $(a=10,000)$ and a short crack ( $a=10)$. (b) The dual model having both micro and macro ligaments, for a crack length $a=200$. 
simplifications such as two-dimensional structures and regular spacings we can investigate systematically whether the TCD can be used to predict behaviour when these mechanisms operate, either individually or in combination.

The above models were investigated separately and then combined in various ways to create multiscale models (known as "dual" models) having features at both micro and macro scales. Four dual models were analysed: (i) micro/macro ligaments; (ii) micro/ macro barriers; (iii) micro-barriers/macro-ligaments and (iv) micro-ligaments/macro-barriers.

In what follows we will use the version of the TCD known as the Point Method (PM); this is the simplest and most commonly used of all the TCD methods. Here, failure is predicted to occur if the stress at a distance $L / 2$ from the crack tip is equal to or greater than a critical value. In many materials (including brittle and quasi-brittle materials and also in the case of metal fatigue) this critical stress is equal to the ultimate strength $\sigma_{\mathrm{u}}$ so this assumption will be used here. However it should be noted that for some materials (especially those displaying a significant amount of plasticity) the critical stress can be higher than $\sigma_{\mathrm{u}}$. In applying the PM to the case of a centre-cracked plate in tension we can make use of the standard Westergaard solution for the stress $\sigma(\mathrm{r})$ as a function of distance $r$ from the crack tip, for a remote applied stress $\sigma$ :

$$
\sigma(r)=\frac{\sigma}{\left[1-\left(\frac{a}{a+r}\right)^{2}\right]^{1 / 2}}
$$

\section{Results}

Fig. 3 shows results from the ligaments models, demonstrating how $K$ changes as the crack extends by $\delta a$. Here $K_{\text {net }}$ is the overall $K$ value found by subtracting the $K$ contribution of each unbroken ligament (using Eq. (4)) from the $K$ value due to the loading which is simply $\sigma \sqrt{ } \pi\left(a_{\mathrm{i}}+\delta a\right)$. In the three examples shown the stress was chosen to correspond to the fracture stress, i.e. the smallest value of $\sigma$ which keeps $K_{n e t}>K_{m}$ and so ensures continued crack propagation. Fig. 3a shows results from the micro model with two different crack lengths. With a long initial crack $\left(a_{\mathrm{i}}=10,000\right), K_{\text {net }}$ decreases monotonically to a value of 0.5 as the crack grows up to $\delta a=40$, due to the recruitment of an increasing number of fibres in the crack wake. Thereafter $K_{\text {net }}$ no longer decreases because further growth causes failure of fibres remote from the crack tip, keeping the process zone size equal to a constant $40 \mathrm{~d}_{\text {micro }}$. If the initial crack is short ( $a_{\mathrm{i}}=10$ in this example) the behavior is quite different: $K_{\text {net }}$ initially increases and then decreases, reflecting the different contributions to $K$ due to crack extension and ligament recruitment, respectively. A minimum is reached at $K_{\text {net }}=0.5$ after which $K_{\text {net }}$ rapidly increases due to the failure of ligaments which reduces the size of the process zone below 40 .

Fig. 3b shows corresponding results for a dual model having both micro and macro ligaments with spacings $d_{\text {micro }}=1$ and $d_{\text {macro }}=100$ and an initial crack length $a_{\mathrm{i}}=200$. Now, two minima occur as the crack extends. The first minimum (at $\delta a=40$ ) is due to the effect of the micro fibres alone since no macro fibres have been encountered at this stage. The second minimum is reached as a result of contributions from both macro and micro fibres: it occurs at $\delta a=3600$ at which point the process zone contains 36 unbroken macro fibres (rather less than the 40 that would have been present in the macro model) and just 2 unbroken micro fibres. The number of micro fibres is small because the applied $\mathrm{K}$ here is much larger than in the micro model, so only micro fibres which are very close to the crack tip remain unbroken. It was found that if the initial crack length is small (less than $a_{\mathrm{i}}=200$ ) then the first minimum is the deepest and so the fracture stress is determined by the behavior of the micro fibres only, but for larger crack lengths the crack arrests at the second minimum and so is affected by the fibres at both length scales.

Fig. 4 shows results for fracture stress as a function of crack length, for the three models which use ligaments: micro, macro and the combined micro/macro dual model. The micro and macro models display behaviour similar to much of the published experimental data (as shown for example in Fig. 1b). The results from the dual model are identical to those of the micro model at small crack lengths and almost identical to the macro model at large crack lengths, though in fact the fracture stress for a given crack length

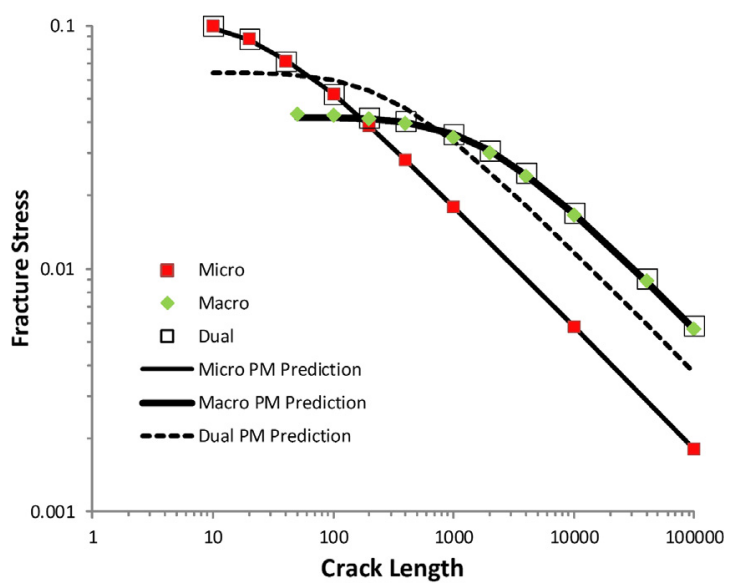

Fig. 4. Results for the three ligaments models, along with predictions using the TCD (Point Method). 
is very slightly higher in the dual model. There is a clear kink in the curve for the dual model around $a_{\mathrm{i}}=200$ coinciding with the change in process zone size mentioned above.

Also included in Fig. 4 are predictions using the TCD (Point Method). For the micro and macro models the TCD gives a very good fit with errors no greater than $2 \%$ in predicting fracture stress. For the dual model the errors are larger, fracture stress is overpredicted by about $30 \%$ at the largest and smallest crack lengths and underpredicted by a similar margin near the kink.

Here an explanation should be given about the exact method used to fit the prediction curve to the data. The error on each point is defined in the normal way as a residual given by the square of the relative difference, so for a predicted value $\sigma_{\mathrm{pi}}$ of a fracture stress point $\sigma_{\mathrm{fi}}$ the residual is given by:

$$
r_{i}=\left(\frac{\sigma_{f i}-\sigma_{p i}}{\sigma_{f i}}\right)^{2}
$$

Normally the best fit would be obtained by minimising the sum of the residuals but this approach gives poor predictions in cases like this because it depends strongly on which data points are available. For example, if (as in this case) more data points are available to the right of the kink point, then the sum (or average) of the residuals is minimised by a curve which passes close to the points at large crack lengths but predicts the small crack points very poorly. So instead the fit criterion used here was to minimise the value of the largest individual residual. This gave a more realistic fitting curve.

Fig. 5 shows the equivalent results for the barrier models. The situation here is somewhat simpler because the crack will either stop at the first micro barrier or at the first macro barrier, since the intervening material is assumed to have a negligible toughness. So for the dual model the results correspond to whichever of the micro or macro predictions gives the highest fracture stress, giving another kinked curve. The accuracy of the various TCD predictions is similar to that for the ligaments models.

Finally, Fig. 6 shows multiscale models having two different toughening mechanisms. A material with micro scale barriers and macro scale ligaments (Fig. 6a) displays a curve with a significant kink around $a_{i}=150$ and the resulting TCD prediction has quite poor accuracy, especially for the shortest and longest cracks. On the other hand, a material with micro ligaments and macro barriers (Fig. 6b) gave rise to a smooth curve with no kink, for which the TCD gives an excellent prediction.

Table 2 shows the values of the critical distance $L$ and other relevant parameters for the various TCD predictions. The predictions were optimised by varying $L$ and the critical strength (material strength $\sigma_{\mathrm{u}}$ ); the resulting $K_{\mathrm{c}}$ value was found using Eq. (6). For the single-scale models the value of $L$ closely reflects the relevant microstructural parameter. For the ligament models $L$ was similar to, though in both cases somewhat smaller than, the process zone size of 40 ligaments, whilst for the barrier models $L$ was almost exactly equal to half the barrier spacing. For the dual models, the resulting value of $L$ is seen to be a compromise between the micro and macro length scales. In these particular examples the micro and macro scales were separated by a factor of 100 and the dual-model $L$ values were approximately a factor of 10 from both scales as a result. For the dual model having micro barriers and macro ligaments, $L$ was again a compromise value reflecting the very different critical distances at the two scales, whilst in the final model, with micro ligaments and macro barriers, the resulting $L=55$ was of the same order of magnitude as both individual values.

\section{Case study: bone}

The above results show that the presence of two toughening mechanisms at different length scales can give rise to a different form of the strength/crack-length curve, having a kink when the dominant mechanism changes, though not in all cases. However these results are for model materials having behaviour which is simplified compared to that of real materials, so it is interesting to consider whether such behaviour really occurs in practice.

One interesting example in this respect is bone. Cortical bone from humans and animals has been tested by a number of workers to

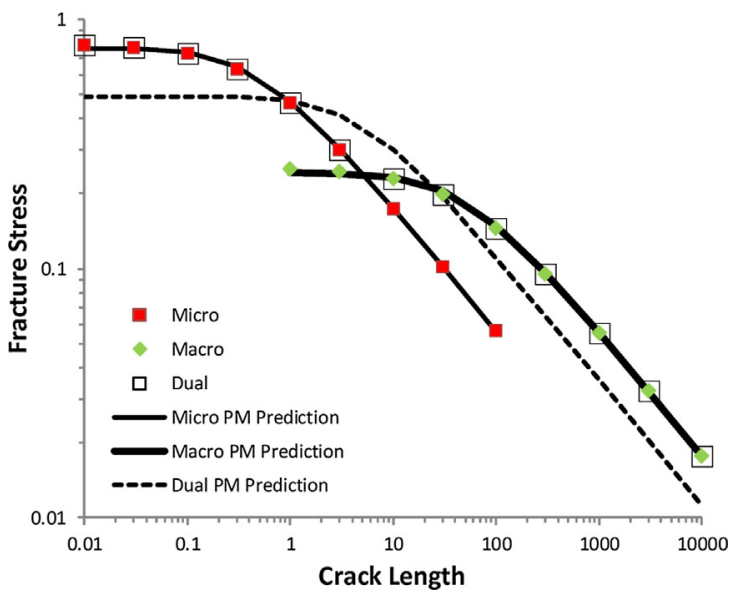

Fig. 5. Results for the three barriers models, along with predictions using the TCD (Point Method). 

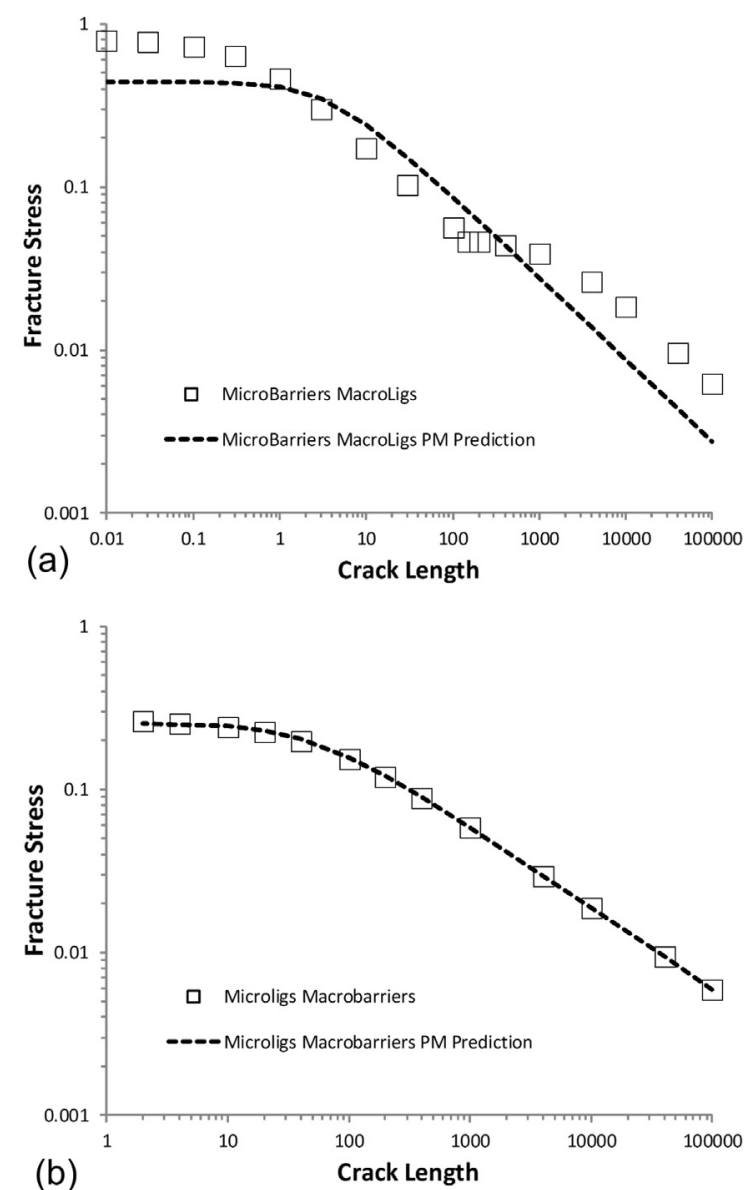

Fig. 6. Results for dual models with mixed toughening mechanisms: (a) micro barriers and macro ligaments; (b) micro ligaments and macro barriers. Predictions using the TCD (Point Method).

Table 2

Constants obtained from the TCD (PM) predictions.

\begin{tabular}{llll}
\hline Model & $\mathrm{L}$ & $\sigma_{\mathrm{u}}$ & $\mathrm{K}_{\mathrm{c}}$ \\
\hline Micro ligaments & 28.8 & 0.107 & 1.02 \\
Macro ligaments & 1820 & 0.0421 & 3.18 \\
Dual ligaments & 340 & 0.0641 & 2.10 \\
Micro barriers & 0.530 & 0.767 & 0.99 \\
Macro barriers & 53.5 & 0.242 & 3.14 \\
Dual barriers & 5.30 & 0.490 & 2.00 \\
Micro-barriers/macro-ligaments & 4.00 & 0.437 & 1.55 \\
Micro-ligaments/macro-barriers & 55.0 & 0.253 & 3.33 \\
\hline
\end{tabular}

show the effect of cracks, notches and holes on its fracture and fatigue behaviour [29-33]. Previously we analysed some of this data using the TCD approach and showed that reasonable predictions of the effects of holes and short cracks could be obtained using a critical distance of $\mathrm{L}=0.38 \mathrm{~mm}$ [34]. This distance does not correspond exactly to any microstructural scale in the material. It is somewhat larger than the size of the largest microstructural units - long fibrous structures called osteons which run longitudinally (i.e. along the long axis of a long bone such as the femur): they are typically 100-200 $\mu \mathrm{m}$ in diameter. But it is known that bone has structure on several smaller length scales: it is essentially a fibre composite laminate material with laminae of thickness about $1 \mu$ m made up of collagen fibres and mineral crystals at the $10 \mathrm{~nm}$ scale. Other workers have noted that bone has several different toughening mechanisms which operate on different length scales [3,35]. This suggests that a simple TCD approach with a single $L$ value will not be the best way to investigate this type of material.

A useful experimental technique in the study of toughening mechanisms is the so-called resistance curve or R-curve, which records the stress intensity required for crack propagation as a function of the amount of crack extension $\delta a$ from an initial sharp notch. Nalla et al. carried out an extensive series of tests to establish R-curves for bone [36,37]. Fig. 7a shows an example of their 

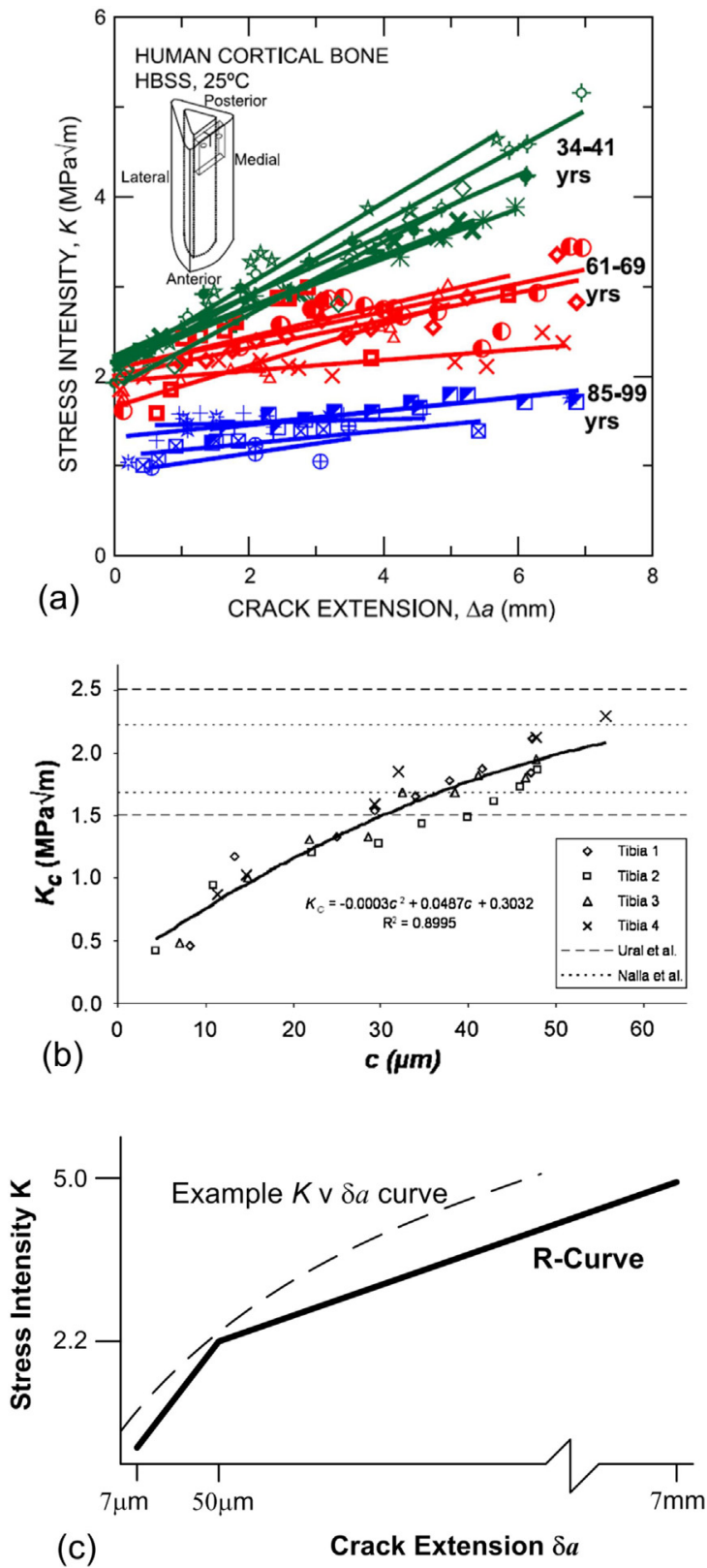

Fig. 7. Experimental R-curves for bone from (a) Nalla et al. [36] and (b) Mullins et al. [38]. (c) An R-curve generated from these two sets of data, used to generate data for bone fracture strength (see Fig. 8).

results from bending tests in which cracks were induced to grow in the longitudinal direction: bone is highly anisotropic so the crack orientation is a crucial factor. Here we will make use of their data from relatively young bones (subjects aged 34-41 years) which show a rising R curve. At the greatest crack extensions studied the stress intensity rose to $K=5 \mathrm{MPa} V \mathrm{~m}$. Normally R-curves level off to a constant value at high $\delta a$ giving the true long-crack toughness $K_{c}$, but this did not happen here. So we will assume here a value of 5 whilst noting that in fact it may be even higher. Broadly similar results have been obtained by other workers [3].

The data of Nalla et al. can be extrapolated to a value of about $2 \mathrm{MPa} \vee \mathrm{m}$ at zero crack extension - the so-called "inititation toughness" $K_{\mathrm{i}}$. Though this parameter is often quoted as a measure of a material's intrinsic toughness, it cannot in fact exist in the general case, because if so then when $a_{\mathrm{i}} \rightarrow 0$ the fracture stress would approach infinity, which is clearly impossible. In the present case there are some other experimental data which demonstrate different behaviour for very short crack extensions. Mullins et al. used an indentation approach, generating cracks from small diamond-tipped indenters [38] and produced data showing the dependence of $K_{\mathrm{c}}$ on crack length for lengths in the range 7-56 $\mu \mathrm{m}$ (see Fig. 7b), These data can also be regarded as an R-curve, because in these types of experiments a crack grows from the indentation and arrests at a certain length when it has insufficient energy to 
propagate further. Indentations were made on the surfaces of samples in such a way that at least one propagation direction was the longitudinal one, so these results can be combined with the data of Nalla et al. to create a single R-curve encompassing the whole range of range extensions from $7 \mu \mathrm{m}$ to $7 \mathrm{~mm}$. As shown in Fig. $7 \mathrm{c}$ this can be approximated with little loss of accuracy as two straight lines intersecting at a value of $\delta a=50 \mu \mathrm{m}, K=2.2 \mathrm{MPa} \vee \mathrm{m}$.

This R curve was used here to generate a strength/crack-length curve for bone as follows. Choosing a given initial crack length $a_{\mathrm{i}}$, and applied stress $\sigma$, one imagines the crack extending. It will continue to extend provided $K$ is greater than the value given by the $\mathrm{R}$ curve: if at any stage $K$ for a given $\delta a$ falls below the line given by the R curve, then the crack will arrest. The predicted fracture stress is the lowest value of $\sigma$ for which no arrest occurs. An example is shown on Fig. 7c. Fig. 8 shows the resulting strength/crack-length plot, which is seen to display kinked behaviour rather like that seen in the model materials above. This figure also shows three other sets of data from different publications in which the fracture toughness of bone was measured using specimens with different initial crack lengths. These data show broad agreement with the results generated from the R-curve though there are some differences. Lakes et al. used short cracks in the range $0.33-0.76 \mathrm{~mm}$ [29]. This data is very valuable because it is the only data from conventional toughness tests for cracks in this size range. However the growth direction for these cracks was transverse, i.e. perpendicular to the longitudinal direction used for all the other tests reported here. Examining results from a wide range of studies (reported by Yan et al. [39]) shows that the fracture toughness in the transverse direction is typically about 2 times higher than in the longitudinal direction, so one can assume that similar tests with longitudinal crack growth would give lower stresses, very close to the results obtained from the R curves. The other two data sets shown on the figure are for conventional fracture toughness tests for longitudinal cracking using longer initial crack lengths $[30,31]$.

Fig. 8 also shows TCD predictions. The best fit to the data from all crack lengths gives a critical distance of $\mathrm{L}=0.35 \mathrm{~mm}$, which is very similar to our value of $0.38 \mathrm{~mm}$ from earlier work [34]. However a much better fit can be obtained by using two different TCD predictions, one for the short cracks and one for the long cracks. The short-crack prediction gives $L=0.065 \mathrm{~mm}$ whilst for the long cracks $L=7.15 \mathrm{~mm}$. This suggests that two different toughening mechanisms are involved, and the examination of other experimental work suggests what these might be. Small cracks are known to arrest when they meet osteons [40,41], suggesting a barrier mechanism. Bone naturally contains many internal cracks which have arrested at lengths of the order of $0.1 \mathrm{~mm}$, suggesting that this is the typical separation of barriers. Other workers have measured the distances between osteon boundaries and found an average value of about $0.12 \mathrm{~mm}[42,43]$. This distance corresponds to $d$ in our model materials with barriers, for which we found $L=0.53 d$, so if osteons were functioning in the same way as the barrier in our model materials we would expect an $L$ value of 0.06 mm, very close to the value found here.

For longer cracks the major toughening mechanism observed is support from osteons acting as uncracked ligaments [35,37]. Whilst the size and spacing of individual osteons is of the order of $0.1-0.2 \mathrm{~mm}$ we know that $L$ can be much larger than $d$ in this case because the process zone can contain many ligaments. For our model materials here we chose 40 ligaments, but this could be changed to any arbitrary number depending on our choice of two parameters: the elastic stiffness and the strain to failure of the ligament material [21]. The L value of $7.15 \mathrm{~mm}$ obtained here suggests something of the order of 30-40 supporting ligaments, similar to the number in our model materials. The fact that the R-curve did not level off in Nalla et al. results suggests that they did not reach the

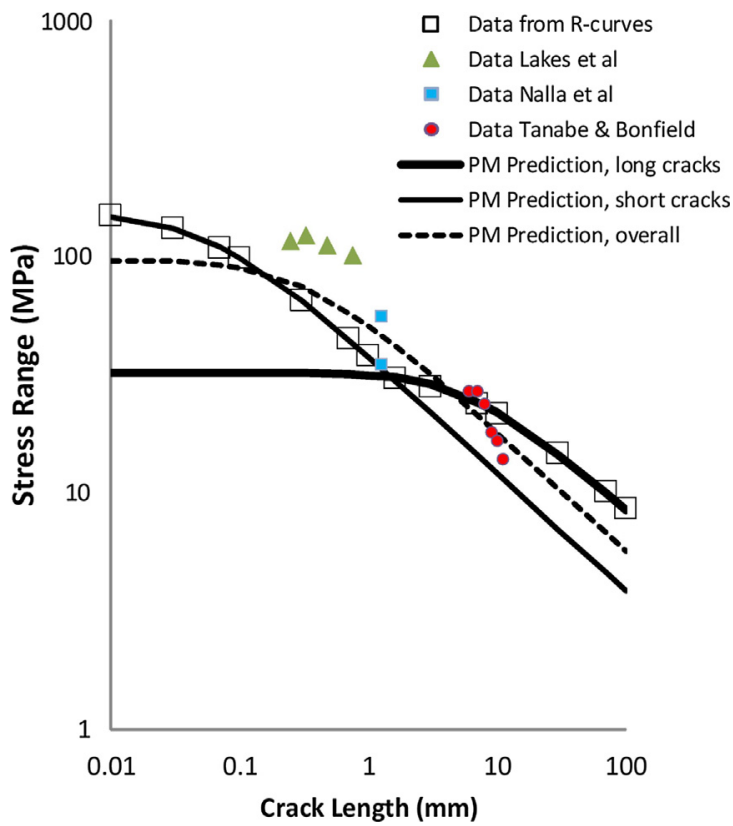

Fig. 8. Experimental data for bone, generated using the R-curves shown in Fig. 7, plus three sets of data from conventional fracture toughness experiments $[29,31,44]$. Three predictions using the TCD (Point Method), based on: the long cracks only, the short cracks only, and an overall prediction using all crack lengths. 
point where a stable process zone size occurred: this is supported by their microscopic observations which showed evidence of unbroken ligaments over the whole crack length. In practice it may not be possible to reach this stable point as it would require quite large specimens.

This case study has shown that it may be possible to detect the existence of multi-scale toughening mechanisms using a more sophisticated version of the TCD approach.

\section{Discussion}

The aim of this work was to investigate the use of the TCD in materials where toughening mechanisms operate on more than one length scale. This was approached using model materials which had previously been shown to give useful results in the simpler case of materials with single toughening mechanisms. In those cases a close correspondence between $L$ and some physical distance was found for the single-scale models, whether micro or macro. This is in agreement with earlier work on these models [20,21] and implies that in these cases the TCD can be used as an analytical tool to shed light on the operative mechanisms.

When two mechanisms were operating at length scales separated by a factor of 100 or more (Figs. 4, 5 and 6a) the TCD approach could still be used and still had reasonable predictive accuracy. Errors as high as $30 \%$ on individual data points occurred, but this would not be unusual for this kind of data which often shows quite a lot of scatter. But the resulting $L$ value had no physical significance, being a compromise value between the larger and smaller scales. However, the existence of a kink in the curve gives a hint that different mechanisms may be operating, and different parts of the curve could then be predicted separately, increasing accuracy and generating physically meaningful $L$ values. This was demonstrated in practice with the example of bone.

These findings reinforce the existing use of the TCD, which is largely employed in a practical manner to predict the effect of stress concentration features in industrial components. In my experience, designers are sometimes concerned about the fact that, when they conduct a TCD analysis, they find an $L$ value which does not seem to relate to any microstructural feature in their material. The present work provides a possible explanation - the material may have multiscale toughening mechanisms - and gives confidence that the TCD can still be used, provided it accurately predicts the available experimental data.

The availability of suitable data is perhaps the main limitation to the practical use of the TCD. It can be difficult and expensive to generate data for a wide range of crack lengths and notch geometries. The result is that TCD parameters obtained from a limited set of data may be employed for use on quite different problems, for example to predict short-crack behaviour when only large notch data is available. The present work shows that significant errors can potentially arise in this way, so whenever possible the test specimens used to find the TCD parameters should have stress concentration features similar in size and shape to those on the components to be analysed.

Another aspect worth mentioning is the nature of the cracks or notches in these specimens. In the present work it was assumed that the initial crack had been introduced in such a way that the toughening mechanisms in the material had not acted on it, and furthermore that the introduction of the crack did not affect the surrounding material. For example, a sharp notch introduced using a machining method which does not create residual stress or heat treatments. A common method to introduce cracks for fracture toughness testing is through cyclic loading: the resulting fatigue crack will be affected by the material's toughening mechanisms, though not necessarily in the same way as a crack propagating under a static load, and so may give rise to different behaviour in the subsequent fracture tests. In most cases it is easier to create a machined notch than a fatigue crack, and there is much evidence to show that provided the notch root radius is less than $L$ it will behave in the same way as a sharp crack anyway.

An interesting finding which emerged incidentally from this work is that in the multiscale (i.e. dual) models in which toughening occurred at two very different scales (Figs. 4, 5 and 6a), only the macro scale contributed significantly to the long-crack toughness. Microscopic features, whether barriers or ligaments, had a negligible effect on the strength of specimens with long initial cracks. The crucial role of the microscopic mechanism in those cases was to raise the performance of the short cracks and thus effectively increase the plain-specimen strength of the material, which in both the ligament and barrier models was increased by about a factor of three compared to the strength of the macro material alone. This was of course due to the particular material constants chosen for these models. So for example the critical $K$ value for the macro barrier was chosen to be larger than that for the micro barrier. However, if we had chosen to make the two critical $K$ values equal, or make the micro one larger, then the macro toughening mechanism would have had no effect at all. Likewise if the macro mechanism were to give a relatively large strength as well as toughness, it will dominate for all crack lengths: this was seen in the final combination studied (see Fig. $6 \mathrm{~b}$ and Table 2). Given that $L$ corresponds to the mid-point of the curve (as shown in Fig. 1a), the macro curve will always be shifted to higher length values than the micro one. The case of the model having micro barriers and macro ligaments (Fig. 6a) is also interesting for another reason. Here, when a long crack was present, the macro ligaments created a large process zone whilst the role of the micro barriers was effectively to increase the material toughness parameter of the material between the ligaments from $K_{\mathrm{m}}=0.5$ to 1.0. This doubling of the small-scale toughness did serve to raise the strength for long cracks, but only by a modest $10 \%$.

Thus, whilst we are only using simplified material models here, the results seem to capture an important truth about the relative roles of toughening mechanisms at different scales in quasi-brittle materials, for which cracking and damage progression is always the principal mode of failure. Such materials would include fibre composites, concretes, wood and bone. Strength can be increased by using mechanisms which hinder crack growth at the nanometer to micron scale, whilst fracture toughness (in the sense of resistance to relatively long cracks) is conferred through mechanisms operating at larger scales, typically from tens of microns to millimetres. The same finding may also be relevant to high cycle fatigue in metals and merits further investigation. 


\section{Conclusions}

1. Model materials can be generated having toughening mechanisms at two different scales. These models display a distinctive relationship between fracture stress and crack length, having a kink in the curve at which the dominant mechanism changes.

2. The Theory of Critical Distances (TCD) is capable of giving reasonable predictions for these dual-mechanism models, but significant errors arise. Improved predictions can be achieved by using a modified approach which captures the contributions of the two different mechanisms.

3. A practical example of the application of this approach is bone. Using experimental data generated from R-curves, two different toughening mechanisms, operating at different length scales, can be identified with the help of this approach.

4. In these models (and in the example of bone) the microscopic toughening mechanism controlled the material's strength but had little effect on its fracture toughness, which instead was controlled by the macroscopic mechanism.

\section{Appendix A. Supplementary material}

Supplementary data to this article can be found online at https://doi.org/10.1016/j.engfracmech.2019.02.012.

\section{References}

[1] Ritchie RO. The conflicts between strength and toughness. Nat Mater 2011;10(11):817-22.

[2] Akkus O, Yeni YN, Wasserman N. Fracture mechanics of cortical bone tissue: a hierarchical perspective. Crit Rev Biomed Eng 2004;32(5-6):379-425.

[3] Vashishth D, Tanner KE, Bonfield W. Experimental validation of a microcracking-based toughening mechanism for cortical bone. J Biomech 2003;36(1):121-4.

[4] Taylor D. The theory of critical distances: a new perspective in fracture mechanics. Oxford, UK: Elsevier; 2007.

[5] Peron M, Torgersen J, Berto F. A novel approach for assessing the fatigue behavior of PEEK in a physiologically relevant environment. Materials 2018:11.

[6] Ibáñez-Gutiérrez FT, Cicero S, Carrascal IA. On the influence of moisture content on the fracture behaviour of notched short glass fibre reinforced polyamide 6. Compos B Eng 2019;159:62-71.

[7] Jenkins A, Fathi E, Belyadi F. Stress field behavior induced by hydraulic fracture in shale reservoirs: a practical view on cluster spacing. J Nat Gas Sci Eng 2017;48:186-96.

[8] Al Zamzami I, Susmel L. On the use of hot-spot stresses, effective notch stresses and the Point Method to estimate lifetime of inclined welds subjected to uniaxial fatigue loading. Int J Fatigue 2018;117:432-49.

[9] Dabiri M, et al. Utilizing the theory of critical distances in conjunction with crystal plasticity for low-cycle notch fatigue analysis of S960 MC high-strength steel. Int J Fatigue 2018;117:257-73.

[10] Kouanga CT, et al. On the estimation of finite lifetime under fretting fatigue loading. Int J Fatigue 2018;112:138-52.

[11] Taylor D, Cornetti P, Pugno N. The fracture mechanics of finite crack extension. Eng Fract Mech 2005;72:1021-38.

[12] Leguillon D. Strength or toughness? A criterion for crack onset at a notch. Eur J Mech A/Solids 2002;21:61-72.

[13] Cornetti P, et al. Finite fracture mechanics: a coupled stress and energy failure criterion. Eng Fract Mech 2006;73(14):2021-33.

[14] Taylor D. The theory of critical distances applied to the prediction of brittle fracture in metallic materials. Struct Integrity Durability 2006;1:145-54.

[15] Ritchie RO, Knott JF, Rice JR. On the relationship between critical tensile stress and fracture toughness in mild steel. J Mech Phys Solids 1973;21:395-410.

[16] Taylor D. Predicting the fracture strength of ceramic materials using the theory of critical distances. Eng Fract Mech 2004;71:2407-16.

[17] Taylor D. Microstructural parameters in the theory of critical distances. Mater Sci Forum 2008;567:23-8.

[18] Whitney JM, Nuismer RJ. Stress fracture criteria for laminated composites containing stress concentrations. J Compos Mater 1974;8:253-65.

[19] Hoey D, Taylor D. Fatigue in porous PMMA: the effect of stress concentrations. Int J Fatigue 2008;30:989-95.

[20] Taylor D. On the role of microstructure in finite fracture mechanics. Procedia Struct Integrity 2016;2:1999-2005.

[21] Taylor D. The theory of critical distances: a link to micromechanisms. Theor Appl Fract Mech 2017;90:228-33.

[22] Kimoto H, Usami S, Miyata H. Relationship between strength and flaw size in glass and polycrystalline ceramics. Japanese Soc Mech Eng 1985;51-471:2482-8.

[23] Usami S, et al. Strength of ceramic materials containing small flaws. Engng Fract Mech 1986;23:745-61.

[24] Awerbuch J, Madhukar MS. Notched strength of composite laminates: predictions and experiments - a review. J Reinf Plast Compos 1985;4:3-159.

[25] El Haddad MH, Smith KN, Topper TH. Fatigue crack propagation of short cracks. J Eng Mater Technol (Trans. ASME) 1979;101:42-6.

[26] Murakami Y. Metal fatigue: effects of small defects and nonmetallic inclusions. Oxford: Elsevier; 2002.

[27] Murakami Y. Stress intensity factors handbook. Oxford, UK: Pergamon Press; 1987.

[28] Janssen M, Zuidema J, Wanhill R. Fracture mechanics. London, UK: Spon Press; 2002.

[29] Lakes RS, et al. Fracture mechanics of bone with short cracks. J Biomech 1990;23:967-75.

[30] Nalla RK, Kinney JH, Ritchie RO. Mechanistic fracture criteria for the failure of human cortical bone. Nat Mater 2003;2:164-8.

[31] Tanabe Y, Bonfield W. Effects of initial crack length and specimen thickness on fracture toughness of compact bone. JSME Int J, Series C: Mech Syst, Mach Elements Manuf 1999;42(3):532-8.

[32] McBroom RJ, Cheal EJ, Hayes WC. Strength reductions from metastatic cortical defects in long bones. J Orthop Res 1988;6(3):369-78.

[33] Edgerton BC, An KN, Morrey BF. Torsional strength reduction due to cortical defects in bone. J Orthop Res 1990;8(6):851-5.

[34] Kasiri S, Taylor D. A critical distance study of stress concentrations in bone. J Biomech 2008;41:603-9.

[35] Zimmermann EA, Ritchie RO. Bone as a structural material. Adv Healthcare Mater 2015;4(9):1287-304.

[36] Nalla RK, et al. Effect of aging on the toughness of human cortical bone: evaluation by R-curves. Bone 2004;35(6):1240-6.

[37] Nalla RK, et al. Mechanistic aspects of fracture and R-curve behavior in human cortical bone. Biomaterials 2005;26(2):217-31.

[38] Mullins LP, Bruzzi MS, McHugh PE. Measurement of the microstructural fracture toughness of cortical bone using indentation fracture. J Biomech 2007; 40(14):3285-8.

[39] Yan J, et al. Fracture toughness of manatee rib and bovine femur using a chevron-notched beam test. J Biomech 2006;39(6):1066-74.

[40] Lee TC, O'Brien FJ, Taylor D. Nature of fatigue damage in bone. Int J Fatigue 2000;22(10):847-53.

[41] O'Brien FJ, Taylor D, Lee TC. The effect of bone microstructure on the initiation and growth of microcracks. J Orthop Res 2005;23(2):475-80.

[42] Burr DB. Bone, exercise and stress fractures. Exerc Sport Sci Rev 1997;25:171-94.

[43] Presbitero G, et al. Distribution of microcrack lengths in bone in vivo and in vitro. J Theor Biol 2012;304:164-71.

[44] Nalla RK, Kinney JH, Ritchie RO. Mechanistic fracture criteria for the failure of human cortical bone. Nat Mater 2003;2(3):164-8. 